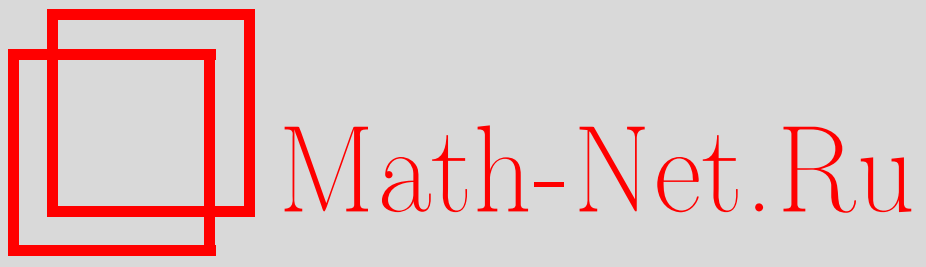

И. Б. Симоненко, Предельные теоремы типа Сегё для многомерных дискретных операторов свертки с непрерывным символом, Функи. анализ и его прил., 2001, том 35, выпуск 1, 91-93

DOI: https://doi.org/10.4213/faa238

Использование Общероссийского математического портала MathNet.Ru подразумевает, что вы прочитали и согласны с пользовательским соглашением

http://www. mathnet.ru/rus/agreement

Параметры загрузки:

IP : 52.87 .193 .239

26 апреля 2023 г., 14:33:48

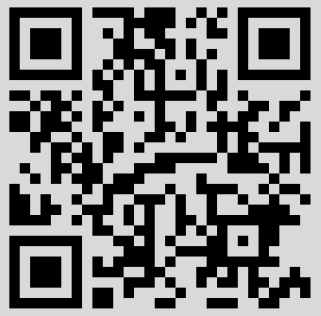


УДК 517.9

\title{
Предельные теоремы типа Сегё для многомерных дискретных операторов свертки с непрерывным символом
}

\author{
(c) 2001. И. Б. Симоненко
}

1. Основные результаты. Пусть число $\operatorname{AvTr}_{f}(A)$, где $A$ - линейный оператор, действующий в конечномерном линейном пространстве размерности $m$, a $f-$ функция, область определения которой содержит в себе спектр оператора $A$, определяется равенством

$$
\operatorname{AvTr}_{f}(A)=\frac{1}{m} \sum_{j=1}^{m} f\left(\lambda_{j}\right)
$$

где $\left\{\lambda_{j}\right\}(j=1, \ldots, m)$ - полный набор собственных чисел оператора $A$ с учетом их кратности. Здесь и всюду далее функция - это комплекснозначное отображение; линейное пространство - это пространство над полем комплексных чисел $\mathbb{C}$. Кроме того, пусть

- $\mathbb{Z}^{n}, n \in \mathbb{N}$, снабжено дискретной мерой, равной 1 на каждом одноэлементном множестве;

- $T=\{z: z \in \mathbb{C},|z|=1\}-$ единичная окружность с лебеговой мерой-длиной $\bar{s}, s=(2 \pi)^{-1} \bar{s}, T^{n}$ снабжено мерой $s^{n}=s \times \cdots \times s(n$ сомножителей $)$;

- $l_{2}=L_{2}\left(\mathbb{Z}^{n}\right), L_{2}=L_{2}\left(T^{n}\right)$ - пространства суммируемых с квадратом функций с естественными для таких пространств нормами;

- fs - множество конечных непустых подмножеств множества $\mathbb{Z}^{n}$;

- $l_{2}(X)$ при $X \in \mathrm{fs}-$ пространство, состоящее из функций, принадлежащих $l_{2}$ и обращающихся в нуль вне $X$;

- $\operatorname{card}(X)$ - число элементов в множестве $X$;

- $\eta$ - функция, определенная на fs следующим образом:

$$
\eta(X)=\inf _{Y \subset X} \max \left(\operatorname{card}(X \backslash Y) / \operatorname{card}(X), 1 / \operatorname{dist}\left(Y, \mathbb{Z}^{n} \backslash X\right)\right),
$$

где $\operatorname{dist}(X, Y)=\inf \{|x-y|: x \in X, y \in Y\},|x|-$ длина вектора $x$;

- $F: l_{2} \rightarrow L_{2}$ - преобразование Лорана:

$$
(F g)(t)=\sum_{\gamma \in \mathbb{Z}^{n}} g(\gamma) t^{\gamma}, \quad \text { где } t^{\gamma}=\left(t_{1}, \ldots, t_{n}\right)^{\left(\gamma_{1}, \ldots, \gamma_{n}\right)}=t_{1}^{\gamma_{1}} \cdots t_{n}^{\gamma_{n}}
$$

- $\operatorname{End}(B)$ - банахова алгебра линейных ограниченных операторов, действующих в В-пространстве $B$;

- $\mathfrak{C}$ - множество тех операторов $A$ из $\operatorname{End}\left(l_{2}\right)$, для которых оператор $F A F^{-1}$ является оператором умножения на некоторую определенную на $T^{n}$ непрерывную функцию; саму эту функцию будем обозначать через $\mu(A)$;

- $A \in \mathfrak{C}, \nu=\mu(A), G$ - выпуклая оболочка множества значений функции $\nu$, $A_{X} \in \operatorname{End}\left(l_{2}(X)\right)$, где $X \in \mathrm{fs},-$ оператор, определяемый равенством $A_{X}=P A j$, в котором $P$ - оператор умножения на характеристическую функцию множества $X$ и $j: l_{2}(X) \subset l_{2}$ (заметим, что для любого $X \subset \mathbb{Z}^{n}$ спектр оператора $A_{X}$ содержится в множестве $G)$; 
- $\Phi_{f}:$ fs $\rightarrow \mathbb{C}$, где $f-$ определенная на $G$ функция, задается формулой

$$
\Phi_{f}(X)=\operatorname{AvTr}_{f}\left(A_{X}\right) .
$$

Теорема 1.1. Если $f$ - функция, определенная и непрерывная на множестве $G$ и аналитическая как функция комплексного переменного во внутренних точках этого множества, то

$$
\Phi_{f}(X) \rightarrow I_{f} \stackrel{\text { def }}{=} \int_{T^{n}} f(\nu) d s^{n}, \quad \kappa о г \partial а \quad \eta(X) \rightarrow 0 .
$$

Далее в этом разделе мы предполагаем, что $\nu$ - вещественная функция, $c=$ $\min \nu$ и $d=\max \nu$.

ТЕОРема 1.2. Пусть $f$ - непрерывная функиия, определенная на сегменте $[c, d]$. Тогда справедливо (1).

Tеорема 1.3. Пусть $Y \subset[c, d]$ u $N(X)$ nрu $X \in$ fs - число собственных чисел оператора $A_{X}$, принадлежащих множеству $Y$, с учетом их кратности. Тогда

$$
s^{n}\left[\nu^{-1}(\operatorname{int}(Y))\right] \leqslant \liminf _{\eta(X) \rightarrow 0} \frac{N(X)}{\operatorname{card}(X)} \leqslant \limsup _{\eta(X) \rightarrow 0} \frac{N(X)}{\operatorname{card}(X)} \leqslant s^{n}\left[\nu^{-1}(\operatorname{cl}(Y))\right],
$$

где $\operatorname{int}(Y)(\operatorname{cl}(Y))$ - внутренняя часть (замыкание) множества $Y$ в сегменте $[c, d]$, а $\nu^{-1}(Z)-$ прообраз множества $Z \subset[c, d]$ при отображении $\nu$.

2. Случай подобно расширяющейся области. Пусть $x_{0} \in \mathbb{R}^{n}, \vartheta_{\alpha}: \mathbb{R}^{n} \rightarrow \mathbb{R}^{n}$ $(\alpha>0)$ - преобразование, определяемое формулой $\vartheta_{\alpha}(x)=x_{0}+\alpha\left(x-x_{0}\right), D$ - ограниченное измеримое по Жордану (см. [1, с. 420, статья «Жордана мера»]) подмножество пространства $\mathbb{R}^{n}$, обладающее положительной мерой, и $D_{\alpha}=\mathbb{Z}^{n} \cap$ $\vartheta_{\alpha}(D)$.

ЛЕмма 2.1. $\eta\left(D_{\alpha}\right) \rightarrow 0$ при $\alpha \rightarrow+\infty$.

Эта лемма позволяет применить теоремы предыдущего раздела в случае подобно расширяющихся областей. Например, следствием теоремы 1.1 является

ТЕОРема 2.1. Если $f$ удовлетворяет условиям теоремы 1.1, то

$$
\Phi_{f}\left(D_{\alpha}\right) \rightarrow I_{f}, \quad \kappa о г д а \alpha \rightarrow+\infty .
$$

3. Комментарии. Интерес автора к данной проблеме возник под влиянием известной статьи [2] (см. также [3, гл. 5]), где был рассмотрен одномерный случай. Наиболее близкой к нашей работе является статья [4], в которой терема 2.1 доказана в предположении, что символ $\nu$ - положительная функция, а $f=\ln$, при более сильных ограничениях на гладкость границы области $D$ и символ: граница области $D$ предполагается $C^{2}$-кусочно-гладкой и обладающей положительной гауссовой кривизной, символ - принадлежащим $W_{2}^{1 / 2}$. Аналогичный результат в континуальном случае получен ранее в [5], где относительно области $D$ предполагается, что она либо является выпуклой, либо представляет собой тело симплициального комплекса. Достаточно полная библиография по этому вопросу содержится в [6, pp. 167-172].

У нас на область $D$ налагается лишь одно условие: она измерима по Жордану и имеет положительный объем, а символ только непрерывен. Отметим, однако, что в [4] и [5] вычислены два члена асимптотики, у нас же - только первый. 


\title{
ЛИТЕРАТУРА
}

1. Математическая энциклопедия. Т. 2, Советская энциклопедия, M, 1979. 2. Szego G. In: Festskrift Marcel Riesz (Lund. 1952), pp. 228-238. 3. Гренандер У., Сегё Г. Теплицевы формы и их приложения. ИЛ, М., 1961. 4. Линник И.Ю. Изв. АН СССР, сер. матем., 39, №6, 1393-1403 (1975). 5. Widom H. Trans. Amer. Math. Soc., 94, No. 1, 170-180 (1960). 6. Böttcher A., Silbermann B. Invertibility and Asymptotics of Toeplitz Matrices. Akademie-Verlag, Berlin, 1983.

Ростовский госуниверситет

Поступило в редакцию 18 мая 1999 г.

УДК 517.3

\section{Дополнение к бифуркационной диаграмме тригонометрических полиномов}

\author{
(c) 2001. С. В. ШАДРИн
}

1. Мы рассматриваем пространство вещественных тригонометрических полиномов $F=\left\{f(t)=\cos n t+\sum_{j=1}^{n-1}\left(A_{j} \cos j t+B_{j} \sin j t\right) \mid A_{j}, B_{j} \in \mathbb{R}\right\}$, определенных на окружности $\left(f: S^{1}=\mathbb{R} / 2 \pi \mathbb{Z} \rightarrow \mathbb{R}\right)$. Бифуркациионной диаграммой $\Sigma_{1} \subset F$ называется множество неморсовских полиномов.

Комплексной формой полинома $f \in F$ мы называем мероморфную функцию $g(z): \overline{\mathbb{C}} \rightarrow \overline{\mathbb{C}}$, получаемую из $f$ заменой $z=e^{i t}$. Обозначим через $\Sigma_{2}$ множество полиномов $f \in F$, комплексные формы которых имеют вещественные непростые критические значения.

Очевидно, что $\Sigma_{1}$ и $\Sigma_{2}-$ гиперповерхности в $F$, причем $\Sigma_{1} \subset \Sigma_{2}$. Статья посвящена описанию дополнений $F \backslash \Sigma_{1}$ и $F \backslash \Sigma_{2}$. Ранее аналогичные вопросы рассматривались в [1] для обычных полиномов и в $[2,3]$ для тригонометрических полиномов с $2 n$ критическими точками на окружности.

2. Функции $f_{1}, f_{2}: S^{1} \rightarrow \mathbb{R}$ называются топологически эквивалентныли, если существуют сохраняющие ориентацию гомеоморфизмы $\alpha: S^{1} \rightarrow S^{1}, \beta: \mathbb{R} \rightarrow \mathbb{R}$, такие, что $f_{1} \alpha=\beta f_{2}$. Очевидно, что если $f \in F$ и $f^{\prime} \in F-$ топологически неэквивалентные морсовские полиномы, то они лежат в разных компонентах дополнения $F \backslash \Sigma_{1}$.

Рассмотрим морсовскую функцию $f_{0}: S^{1} \rightarrow \mathbb{R}$. Обозначим через $\left[f_{0}\right]$ подмножество в $F$, состоящее из всех морсовских полиномов, топологически эквивалентных $f_{0}$. Пусть число критических точек функции $f_{0}$ на окружности равно $2 k$. Очевидно, что если $k>n$, то $\left[f_{0}\right]$ пусто. Поэтому в дальнейшем мы во всех рассуждениях будем предполагать, что $k \leqslant n$.

ТЕОремА 1. Для любой морсовской функции $f_{0}$, такой, что $k \leqslant n$, множество $\left[f_{0}\right]$ непусто. Кроме того, если $k<n$, то $\left[f_{0}\right]-$ связное подмногообразие в $F$. Если же $k=n$, то $\left[f_{0}\right]-$ подмногообразие в $F$, гомотопически эквивалентное $n$ точкам.

Эта теорема дает описание всех компонент дополнения $F \backslash \Sigma_{1}$.

3. Скобочным символом называется синтаксически правильная последовательность из $2 k$ пар квадратных скобок, идущих подряд, и $n-k$ пар круглых скобок, 